subject of a further chapter. Under the heading, "The external milieu", the role of foreign pressure in South African policy making is analysed.

Geldenhuys provides a wealth of new and fascinating information, a good deal of it obtained through personal interviews with South African politicians and officials. Dealing with such a contemporary subject and given the South African government's obsession with secrecy on so many features of policy making, the book inevitably contains many conjectural, tentative and speculative elements. The last word on the formation of South African foreign policy has certainly not been written. Geldenhuys nonetheless provides a rare glimpse behind the well-hidden corridors of power in South Africa. He handles his subject matter in a generally detatched scholarly fashion, thus avoiding the ideological and political pitfalls resulting from any "committed" perspective.

Foreign readers will be particularly interested in the sections dealing with the activities abroad of South Africa's former Department of Information, the Republic's involvement in the Angolan war in 1975-76, the influence of foreign pressure on the country's foreign and domestic policies, and also in the role of the much heard State Security Council in policy making. As regards South Africa's intervention in Angola, for example, the author provides us with a good deal of hitherto unknown information on how and why the Republic got embroiled in the war.

For those taking a more academic interest in the making of foreign policy, Geldenhuys' book has a concluding chapter providing some theoretical perspectives on foreign policy formation and also proposing the outlines of a model of South African foreign policy making.

Finally, the book contains a number of tables and charts, detailed footnotes and a comprehensive index.

Klaus von der Ropp

\title{
Friedrich-Naumann-Stiftung (Hrsg.)
}

\section{South-Africa - a Chance for Liberalism?}

Papers presented during a Seminar of the Friedrich Naumann Foundation in December 1983, Sankt Augustin, Liberal Verlag, 1985, 416 S., DM 38,-

Verfahren, hoffnungslos, einer unaufhaltsamen Polarisierung zustrebend - so wollen viele Beobachter die Lage in Südafrika bezeichnet wissen. Zu differenzierenderen Betrachtungen werden weder die weißen noch die schwarzen Südafrikaner von vielen für fähig gehalten, die Gegensätze scheinen deterministisch einer Eskalation entgegenzustreben. Dieses Negativszenario, von den westlichen Medien genüßlich aufbereitet, vermittelt in seiner Ausweglosigkeit weder einen Ansatz zu einer friedlichen Veränderung, noch wird es der tatsächlichen Lage im Innern Südafrikas gerecht. 
Die Reduktion der politischen Landschaft im Apartheidstaat auf die pointierte, gleichwohl fehlerhafte Einteilung in schwarze Freiheitskämpfer versus weiße Rassisten wird gerade auch dann zerbrochen, wenn der offene Blick die Landschaft der weißen Politiker differenzierter zu fassen sucht. Mit der Föderalen Fortschrittspartei (Progressive Federal Party, PFP), die bei den Wahlen 1981 circa zwanzig Prozent der weißen Wählerstimmen erhielt und mit 26 von insgesamt 165 Parlamentariern die wichtigste weiße Oppositionspartei bildet, stellt sich die liberale weißafrikanische Mindestposition zum Apartheid-Regime wie auch zur schlichten Machtübergabe an die schwarze Bevölkerungsmehrheit nach dem Verdikt des "One man, one vote". Die PFP ist in den letzten Jahren zu einem wichtigen Hoffnungsträger für einen friedlichen Wandel geworden, Ausdruck der weißen Opposition zum Apartheid-System ebenso wie Gegner einer Kapitulation der sich mit gutem Grund als Afrikaner fühlenden Weißen des Landes.

Die Unterstützung der PFP im Ausland erwies sich bisher als wichtiger Faktor zur innenpolitischen Konsolidierung und Stabilisierung der aus der desintegrierten United Party - der letzten regierenden weißen Partei vor dem Machtantritt der Nationalen Partei 1948 - erwachsenen Gruppierung unter ihrem dynamischen und nachdenklichen Vorsitzenden Frederick van Zyl Slabbert. In der Bundesrepublik Deutschland verfügt die PFP über vielfältige Gesprächskontakte, am ausgeprägtesten zur F.D.P. Sichtbarster Ausdruck war ein Seminar in der Friedrich-Naumann-Stiftung im Dezember 1983, an dem vierzehn PFP-Abgeordnete teilnahmen und dessen Ergebnisse nun in ansprechender Form vorliegen.

In kenntnisreicher und respektheischender Weise entwerfen die als Tagungsredner in die Bundesrepublik Deutschland gereisten PFP-Abgeordneten das Bild ihres zerrissenen Landes und Bausteine einer Zukunft, die vom Apartheid-System Abstand nimmt und doch zugleich die Minderheitenrechte der weißen Südafrikaner zu sichern vermag. In seinem eigenen Beitrag führt PFP-Vorsitzender Slabbert die zentralen Positionen der liberalen Hoffnung Südafrikas auf: "To combat racial polarisation, to broaden the constitutional debate, to question the actions of Government, to defend and extend civil rights, to oppose the policy of Apartheid or separate development in all its forms, to propose an alternative constitutional solution" (S. $42 \mathrm{f}$.).

Die Beiträge der PFP-Abgeordneten fügen sich zu einem Reader zusammen, der in abgewogener, kundiger und zugleich engagierter Weise das Porträt Südafrikas und seiner brennenden politischen Probleme zeichnet, ohne in Fatalismus oder Extremismus zu verfallen. Eingerahmt werden diese authentischen Stimmen einer liberalen weißen Alternative zum derzeit regierenden Regime in Pretoria durch Beiträge von drei der kompetentesten Kenner Südafrikas in der Bundesrepublik Deutschland: Reinhard Erkens stellt in seinem Essay die Geschichte des politischen Liberalismus in Südafrika dar, um Möglichkeiten und Grenzen, vor allem aber die faktische Gegebenheit dieser politischen Alternative aufzuzeigen. Theodor Hanf warnt davor, daß die anhaltende Gewalt zu keinem Sieg führen könne und damit für alle Beteiligten umso tragischer bleiben müsse; engagiert setzt er - Autor einer weite Beachtung gefundenen Studie über die Möglichkeiten friedlichen Wandels in Südafrika - sich für die Lösung einer "consociational rule" ein 
nach dem Vorbild der Schweiz, Ósterreichs, des Libanon und Jugoslawiens. Klaus Baron von der Ropp schließlich erneuert seine vieldiskutierte Perspektive einer Teilung Südafrikas, falls alle anderen Lösungsmöglichkeiten scheitern sollten, favorisiert hingegen die Position der PFP und ihres Eintretens für ein bisher unbekanntes Modell mit gleichen Rechten und speziellem Minderheitenschutz für die Weißen. Die Existenz der Föderalen Fortschrittspartei unterstreicht, daß Südafrika eine Herausforderung gerade auch an den Westen bleibt, die als Antwort mehr als nur schablonenhafte Stereotypen verlangt. Das vorliegende Buch setzt hinter dieses Anliegen ein großes Ausrufezeichen.

Ludger Kühnhardt

\section{Winrich Kühne}

\section{Südafrika und seine Nachbarn: Durchbruch zum Frieden?}

Zur Bedeutung der Vereinbarungen mit Mozambique und Angola vom Frühjahr 1984. Aktuelle Materialien zur Internationalen Politik, Stiftung Wissenschaft und Politik/ SWP, Band 7, Nomos-Verlagsgesellschaft, Baden-Baden, 1985, 166 S., DM 22.-

Die südafrikanische Vertragspolitik wurde von ihren Protagonisten enthusiastisch als Beginn eines goldenen Zeitalters der Freundschaft und Zusammenarbeit in einer ehemals konfliktzerrissenen Region begrüßt. Kritiker der neuen Entspannungspolitik Südafrikas dagegen haben von Anfang an mit Skepsis reagiert. Weder sei diese Politik neu, noch diene sie der Entspannung.

Winrich Kühne zählt zu diesen Kritikern. Die zentrale These seiner Arbeit lautet: Die regionale Außenpolitik Pretorias ist Reflex der innergesellschaftlichen Konflikte im Apartheid-Staat. Ziel dieser Politik ist die Schaffung eines cordon sanitaire aus schwachen Satellitenstaaten, die dem exilierten Afrikanischen Nationalkongreß die Aufmarschbasen entziehen sollen.

Vor die Wahl gestellt, das Apartheidsystem abzubauen oder es in der Fläche zu verteidigen, hat sich Pretoria für das letztere entschieden. Diese regionale Anti-Guerilla-Strategie operiert mit allen denkbaren (und undenkbaren) Mitteln, die einer regionalen Vormacht zur Verfügung stehen: Wirtschaftlicher Druck, politische Pressionen und militärische Destabilisierung. Die südafrikanische Regionalstrategie fand ihren vorläufigen Höhepunkt in der "Waffenstillstands- und Rückzugsvereinbarung" mit Angola vom 16. 2. 1984 und im Abkommen über "Nicht-Angriff und gute Nachbarschaft" (sogenannter Vertrag von Nkomati) mit Mozambique vom 16. 3. 1984. Diese Verträge sind nach Kühne keine Wende der südafrikanischen Außenpolitik, sondern weitere Stationen einer Sicherheitspolitik zur Verteidigung des weißen Minderheitsregimes. Die Ursachen dieser Verträge unter Ungleichen waren die Wirtschaftskrisen in Angola und Mozambique, sozialistisches Mißmanagement und die militärischen Interventionen Südafrikas. Sie zwangen die Volksrepubliken an den Verhandlungstisch. Beide Verträge kamen unter 